CERN-TH.6889/93

\title{
Quantum mechanics, common sense and the black hole information paradox
}

\author{
Ulf H. Danielsson and Marcelo Schiffer" \\ Theory Division, CERN, CH-1211 Geneva 23, Switzerland.
}

\begin{abstract}
The purpose of this paper is to analyse, in the light of information theory and with the arsenal of (elementary) quantum mechanics (EPR correlations, copying machines, teleportation, mixing produced in sub-systems owing to a trace operation, etc.) the scenarios available on the market to resolve the so-called black-hole information paradox. We shall conclude that the only plausible ones are those where either the unitary evolution of quantum mechanics is given up, in which information leaks continuously in the course of black-hole evaporation through non-local processes, or those in which the world is polluted by an infinite number of meta-stable remnants.
\end{abstract}

\footnotetext{
${ }^{1}$ Supported by a John Stewart Bell Fellowship
} 
CERN-TH.6889/93

May 1993 


\section{Introduction}

In this paper we will discuss the black-hole information paradox, first discovered by Hawking [1]. As discussed by him, when a pure state has collapsed to form a black hole, it will later evolve into a mixed one as the outcome of the complete evaporation of the black hole. In the wake of this observation, a fierce controversy emerged in the literature. 't Hooft [2] proposed, as a way out of the paradox, that some unknown mechanism could provide the needed correlation between incoming and outgoing radiation to save the unitary evolution of quantum states. Nevertheless, as became increasingly clear during the past year or so, a resolution of the paradox will need a much better understanding of the interplay between gravity and quantum mechanics than is currently at hand. In this context, a lot has been learnt from studies of two-dimensional black holes initiated by Callan et al. in [3]. It might even be that the information paradox is our best clue to the elusive quantum gravity theory. It is therefore of extreme importance to have a thorough understanding of this paradox, as free of model-dependent technicalities as possible.

With the arsenary of elementary quantum mechanics and some information theory we will illustrate the paradox. Our simple analysis will shed some light on the very nature of the paradox and define the properties that any

solution must possess. In particular, we will consider the point of view that a black hole is a "quantum object", somehow implying that our usual intuition of what is wrong and right in physics is not applicable. This typically suggests that EPR-like correlations are important and that this would circumvene the standard arguments leading to the paradox. We will find that no such easy way out is possible.

We will begin by analysing the problem using elementary quantum mechanics. Then, in section 3, we will use information theory to derive some simple results concerning the way in which information may be stored in black holes.

In section 4 we will arrive at a standard set of possibilities, however with a much better understanding of why none of these can be conservative in the sense of not involving fundamentally new phenomena. 


\section{Quantum Copy Rights}

In this section we will consider limitations on the possible resolutions of the information paradox due to quantum mechanics. It is important to see why certain obvious suggestions do not work.

We will consider a situation where the information is "copied" before the in-falling matter crosses the horizon. In this way it is made available to the Hawking radiation. In fact, for an outside observer, the in-falling matter will not be seen to cross the horizon until very late. For an eternal black hole it would never be seen to cross. Hence one might think that all information is conveniently stored and accessible. Still, the act of making a copy is necessary if the original is assumed to continue through the horizon and into the black hole. This, in turn, is based on our expectation, due to the equivalence principle, that the horizon does not have any exceptional local properties capable of completely reflecting all information. If this had been the case we would have had a very simple resolution of the paradox at hand.

In general, both the original and the copy may experience a unitary transformation through some scattering matrices. The process is schematically

$$
|\psi\rangle \rightarrow\left|\psi_{B}\right\rangle \otimes\left|\psi_{O}\right\rangle
$$

Since the final state is a direct product between the internal black- hole state $\left|\psi_{B}\right\rangle$ and the outside state $\left|\psi_{O}\right\rangle$, there are no correlations between the inside and the outside. Hence, if we ignore the inside, i.e. take the trace, no mixing will result on the outside. There would then be no loss of information. Is this a possible scenario? Unfortunately (1) is forbidden. One cannot copy quantum states in this way [4]. The proof goes as follows. Let us assume the state to be copied to be a spin $1 / 2$ particle with states $|\downarrow\rangle$ and $|\uparrow\rangle$. For simplicity we will ignore the state of the copying machine itself. This can be taken into account, 四, with no change in the conclusions. The copying process must be described by some unitary operator $U$. Let us assume that the copying process works for states that are purely up or down. By linearity we then have

$$
U[(a|\downarrow\rangle+b|\uparrow\rangle]=a|\downarrow\rangle|\downarrow\rangle+b|\uparrow\rangle|\uparrow\rangle .
$$

However, the desired state

$$
(a|\downarrow\rangle+b|\uparrow\rangle)(a|\downarrow\rangle+b|\uparrow\rangle)=a^{2}|\downarrow\rangle|\downarrow\rangle+b^{2}|\uparrow\rangle|\uparrow\rangle+a b(|\downarrow\rangle|\uparrow\rangle+|\uparrow\rangle|\downarrow\rangle)
$$


cannot result for general $a$ and $b$, since $U$ produces no states $|\downarrow\rangle|\uparrow\rangle$ or $|\uparrow\rangle|\downarrow\rangle$. We conclude that even if one can construct a $U$ which works for a given state, the same $U$ will not work for all states. In a sense, $U$ is too good at making copies! The correlations are always perfect in the up/down basis. Hence taking the trace over one subsystem produces a maximal mixing in the other subsystem and hence a loss of information. In fact, in this case all information is stored in the correlations.

Now, can this perfect correlation be exploited? If we, given the outside state, always know the inside through these perfect correlations, clearly there can not be any loss of information. It would be silly to take the trace over the inside, since it is identical to the outside, and interpret this as true entropy. The situation recalls of the EPR-phenomenon. Is this the way to solve the paradox? Again the suggestion does not work. The reason is that the correlation cannot be perfect for all states in all bases. This is clearly needed if we are allowed to make any measurement that we want. Consideronce more our example:

$$
U(a|\downarrow\rangle+b|\uparrow\rangle)=a|\downarrow\rangle|\downarrow\rangle+b|\uparrow\rangle|\uparrow\rangle .
$$

Use

$$
|\downarrow\rangle=\frac{1}{\sqrt{2}}(|\rightarrow\rangle-|\leftarrow\rangle) \quad,|\uparrow\rangle=\frac{1}{\sqrt{2}}(|\rightarrow\rangle+|\leftarrow\rangle)
$$

to get

$$
\begin{gathered}
a|\downarrow\rangle|\downarrow\rangle+b|\uparrow\rangle|\uparrow\rangle \\
=\frac{a+b}{2}(|\rightarrow\rangle|\rightarrow\rangle+|\leftarrow\rangle|\leftarrow\rangle)+\frac{a-b}{2}(|\rightarrow\rangle|\leftarrow\rangle+|\leftarrow\rangle|\rightarrow\rangle) .
\end{gathered}
$$

It is only when $(a=-b) a=b$ that the (anti-) correlation is perfect. In the EPR case this means that it is only for singlet states that the anti-correlation is perfect in all bases. Hence, since the correlation is not perfect in general, we are forced to take the trace. At any rate, for a given unknown state, an EPR- related state cannot be obtained through a unitary copying process that works for a general state.

For completeness we should note one loophole in the above argument. This is the case of black-hole hair as discussed in [5]. According to these ideas there are an infinite number of conserved quantities in the world whoose conservation protects unitarity. For this to be the case, everything needs to be conserved, which amounts to say that the world is an integrable system. 
This means that there are superselection rules that forbid superpositions. Compare the superselection rule for electric charge. In the presence of these superselection rules the above argument will not hold. On the other hand, one faces the difficult problem of reconstructing quantum mechanics as we know it, starting with this barren universe.

It seems, therefore, that we have to cope with the fact that information does cross the horizon and is at least temporarily hidden from the outside observer. The questions then are: if, when and how is the information restored? In the next section we will consider the possibility that the information is stored not locally, in the black hole, but rather in its correlations with the environment.

\section{How to Store Information}

As is well known, there is a fundamental objection from QFT to the idea that the information is stored in a black-hole remnant. Low-mass objects with a huge number of internal states would suffer from enormous production rates completely inconsistent with observations. This argument is not qualitatively changed if we take into account that the remnants may slowly evaporate and disappear. Since very little energy is available and a lot of information must be transmitted, the needed time is very long and the remnant effectively stable as far as the argument is concerned [6].

There have been attempts to construct remnants that would not have this defect [7]. However, these attempts seem to run into inevitable problems [8]. We will not consider this further.

In an interesting paper [9], it has been suggested that the information need not be stored locally in the remnant, which implies the above problem, but rather in its correlations with the outside world. This would then, it seems, point at a conservative resolution of the paradox. It is important to note that the correlations we have in mind are correlations between the emitted radiation and the black hole, not correlations between radiation emitted at different times. The reason that the latter is not so relevant is that until the late-time radiation is emitted, the information still has to reside somewhere. This must be inside the black hole. This is because, as we proved in the previous section, given some reasonable assumptions, there will always be information crossing the horizon that is impossible for the Hawking radiation 
to copy. As we will see, and comment on later on, the correlations can be restored to the Hawking radiation (e.g. between radiation emitted at different times) only through non-local processes.

Below, we will analyse the situation using information theory. We will consider two coupled systems 1 and 2 with basis $|n\rangle_{1}, n=1, \ldots, N_{1}$, and $|m\rangle_{2}$, $m=1, \ldots, N_{2}$, where $N_{2} \geq N_{1}$. We will assume that the initial state of the combined system is pure, i.e.that

$$
|\psi\rangle=\sum_{n, m=1}^{N_{1}, N_{2}} A_{n m}|n\rangle_{1}|m\rangle_{2} .
$$

The corresponding pure density matrix is

$$
\rho=\sum_{n, m, k, l}^{N_{1}, N_{2}} A_{n m}|n\rangle_{1}|m\rangle_{21}\left\langle\left. p\right|_{2}\langle q| A_{p q}^{*} .\right.
$$

From this one may construct reduced, in general mixed, density matrices for the individual subsystems 1 and 2 . For 1 we obtain

$$
\rho_{1}=\sum_{j, n, p}^{N_{1}, N_{2}} A_{n j} A_{p j}^{*}|n\rangle_{11}\langle p|
$$

and for 2 we get

$$
\rho_{2}=\sum_{j, m, q}^{N_{1}, N_{2}} A_{j m} A_{j q}^{*}|m\rangle_{22}\langle q| .
$$

Information will be defined as follows [10

$$
I=I_{\max }+\operatorname{Tr} \rho \log \rho
$$

where $S=-\operatorname{Tr} \rho \log \rho$ and $I_{\max }=S_{\max }$. The entropy, $S$, is to be thought of as a lack of information. Note that $S=0 \Rightarrow I=I_{\max }$ and $S=S_{\max } \Rightarrow I=$ 0 . If the number of states is $N$, we have $S_{\max }=-N \frac{1}{N} \log \frac{1}{N}=\log N$, where $\rho=\frac{1}{N}$ for all states. So,

$$
I=\log N+\operatorname{Tr} \rho \log \rho .
$$

With two subsystems we have

$$
I_{1}=\log N_{1}+\operatorname{Tr} \rho_{1} \log \rho_{1},
$$




$$
\begin{aligned}
I_{2} & =\log N_{2}+\operatorname{Tr} \rho_{2} \log \rho_{2}, \\
I_{\text {tot }} & =\log N_{1} N_{2}+\operatorname{Tr} \rho \log \rho,
\end{aligned}
$$

and

$$
I_{\text {tot }}=I_{1}+I_{2}+I_{12},
$$

which defines $I_{12}$, the information content of the correlations.

With a pure total state the total information is maximized (i.e. the entropy is zero)

$$
I_{\text {tot }}=\log N_{1}+\log N_{2} .
$$

What then can be said about the information content of the separate systems 1 and 2? Clearly $I_{1, \max }=\log N_{1}$ and $I_{2, \max }=\log N_{2}$, but what else can we know? Below we will prove that

$$
I_{2, \min }=\log N_{2}-\log N_{1} .
$$

The proof is simple: $A_{n m}$ is an $N_{1} \times N_{2}$ matrix ( $N_{1}$ rows and $N_{2}$ columns); $\rho_{1}=A A^{\dagger}$ is an $N_{1} \times N_{1}$ matrix and $\rho_{2}=\left(A^{\dagger} A\right)^{*}$ an $N_{2} \times N_{2}$. We first prove that $A^{\dagger} A$ has at least $N_{2}-N_{1}$ zero eigenvalues. To do so, let us construct the $N_{2} \times N_{2}$ matrix $\tilde{A}$ by adding $N_{2}-N_{1}$ rows of zeros. Clearly $A^{\dagger} A=\tilde{A}^{\dagger} \tilde{A}$ and $\tilde{A}$ has then at least $N_{2}-N_{1}$ zero eigenvalues by construction. If $\tilde{A}$ is diagonalized, so is $\tilde{A}^{\dagger} \tilde{A}$. Therefore we find that $\tilde{A}^{\dagger} \tilde{A}$, and also $A^{\dagger} A$, have at least $N_{2}-N_{1}$ zero eigenvalues. To minimize $I_{2}$ we must put $\rho_{2}=\frac{1}{N_{1}}$ for the remaining $N_{2}-\left(N_{2}-N_{1}\right)=N_{1}$ non-zero eigenvalues. Then (16) follows.

The result (16) is very reasonable. A little tracing in a small subsystem cannot produce a lot of entropy, or loss of information, in the rest of the system.

Let us now pretend that system 2 is the outside world, containing the Hawking radiation, and that system 1 is the interior of the black hole. If we find that there is very little information in 2, i.e. $I_{2} \sim 0$, we must conclude that $N_{1} \sim N_{2}$. That is, the number of internal states must be very large. It might, however, still be the case that the information is not stored in system 1 but in the correlations, i.e. $I_{1}=0$ and $I_{12} \neq 0$. The important point is that if the information is to be stored in the correlations between the subsystems, each of the subsystems must still have the capacity to store (half of) the information. This must be the case even if the capacity is not used!

Let us now be more precise and relate the above reasoning to a more realistic model of a black hole. When the black hole is formed, we assume 
that the total system is in a pure state. There is information stored in the outside world, the black hole itself, and necessarily also in correlations. The latter is a consequence of the non-existence of perfect copying machines, as we saw in the previous section. As the black hole begins to evaporate, entropy will be produced in the outside world subsystem. Our objective is to estimate a lower limit on this entropy if we ignore back reaction or any other transfer of information to the Hawking radiation. The total entropy carried by the radiation per unit time during the evaporation is then

$$
\dot{S}=\sum_{j} \int \frac{d \omega}{2 \pi} S_{j}(\omega),
$$

where $d \omega / 2 \pi$ is the number of phase cells per unit time that emanate from the black hole and $S_{j}$ is the entropy in a given field mode of the j-th species [11]

$$
S_{j}(\omega)=-\left[\bar{n}_{j} \ln \bar{n}_{j} \mp\left(1 \pm \bar{n}_{j}\right) \ln \left(1 \pm \bar{n}_{j}\right)\right] .
$$

Here and in what follows, the lower and upper signs apply for fermions and bosons, respectively. On the other hand, the mean number of quanta emitted in a given mode by the back hole is [1]:

$$
\bar{n}=\frac{\Gamma}{e^{x} \mp 1},
$$

with $x=\hbar \omega / T_{\mathrm{bh}}$ and $\Gamma$ is the black hole absorptivity.

The calculation of the entropy flux in eq. (17) by means of the above equations has to be carried out numerically, because the black-hole absorption coefficient cannot be cast in a closed form. Here, we borrow Page's [12, 13] result where he calculated $\dot{S}$ numerically for a mixture of three species of neutrinos and antineutrinos, photons and gravitons

$$
\dot{S}=1.619 \frac{\dot{E}}{T_{\mathrm{bh}}} .
$$

Integrating this equation, we obtain the amount of mixing in the radiation produced along the black-hole history. Together with eqs. (13 and 16) we can write the relations

$$
\ln N_{1}>S_{\text {radiation }}=1.619 S_{\mathrm{bh}}
$$


So, the presence of entropy in the outside world puts a lower limit on the number of necessary states of the black hole. Note that this really is a lower limit: there is also entropy initially, before the evaporation has begun, which is due to the always present correlations between what went in and what stayed behind. This may generally be of the same order.

These relations teach us two things. First, if the information has not been returned through Hawking radiation as the black hole approaches the Planck mass, then the remnant has to have an enormous number of internal states to save unitarity. The information might be stored in correlations, as in [9], but this does not solve the remnant problem. Secondly, if we decide to follow the rules of quantum mechanics, we have to seriously interpret $e^{S_{\mathrm{bh}}}$ as the number of black-hole quantum states. The black hole must make full use of its quantum states in order for the information that it subtracted from the enviroment to be momentarily stored either in these states themselves or in correlations. Furthermore, we have learned from the previous discussion the information in question cannot wait until the last moments of evaporation to be restored. Accordingly, it has to leak steadily in the course of black-hole evaporation.

A popular point of view is that back reaction could transfer the information from the in-falling matter forming the black hole to the Hawking radiation. As we have seen, there are two sources of entropy for the outside world. One is the matter that formed the black hole, the other one is the Hawking radiation, or rather the negative energy part that falls into the black-hole. The idea of back reaction suggests that the Hawking pair production is influenced, in such a way that the two potential sources of entropy conspire so that at the end no entropy is produced. As we have seen in the previous section, such a process can never be perfect, if, as is commonly assumed, it is possible to travel into a black hole without losing one's memory. In this connection, it has recently been shown that stimulated emission (bosons) and the exclusion principle (fermions) are two such mechanisms, providing an imperfect correlation between incoming and outgoing radiations, which allows a partial transfer of the information content of the former to the latter [14]. Hence, the only remaining possibility is non-local information transfer. 


\section{Three Possibilities}

In view of the previous discussion, we see only three possible solutions to the paradox.

I. Give up unitary quantum mechanics.

II. Find a way to get along with the remnants. No such possibility seems to exist at the moment 8$]$.

III. The information is restored as the black hole evaporates. This requires non-local effects.

We will discuss the third possibility in a little more detail. The nonlocality which is needed is not just the standard non-locality of quantum mechanics. This would have been in the spirit of correlations, and we have just shown that this is not enough. Instead, one needs a rue information flow from behind the (apparent) horizon.

It is amusing to compare this situation with the idea of Bennet et al [15] on teleportation. There a state is destroyed at one point in space time only to reappear at another. Two kinds of information transfer are needed: one nonlocal EPR-like piece and one classical piece, which must respect the causal structure of space-time. More precisely, the sender and the receiver are each equipped with the members of EPR pairs. The sender brings its EPR particles together with the state to be teleportated. He then makes some measurements on the combined system. The results are then sent to the receiver who, with this knowledge, may reconstruct the teleportated state. This is also the case here. In fact, the parallel is rather complete. The EPR pairs are the pair-produced Hawking radiation, with one particle escaping and the other one venturing into the black hole. The problem is that the second part of the information transfer, which is crucial as we have seen, is troubled by the horizon. Now, the relevant horizon is an apparent horizon, which means that escape is possible but has to be delayed until very late. At this later stage the storage capacity of the black hole has necessarily decreased, unless we contemplate alternative II. Therefore the information must either be destroyed, alternative $\mathbf{I}$, or transferred from the interior and the correlations to the exterior, alternative III.

In the latter case, the question is how? If we trust the correspondence principle, no spectacular quantum gravity effects could occur in the outgoing radiation when the black hole is large with respect to Planckian scales. So it seems that the black-hole must make use of nonlocal effects through its 
quantum states for transferring the information in question.

It has been recently suggested [16, 17], based on information theoretic premisses, that the black-hole event horizon is quantized in units of Planck length squared and, furthermore, similarly to what happens in atomic physics, the leakage of information is made possible by transitions among various quantum black-hole states (black hole-spectroscopy) [17]. Let us analyse, from the information theoretic point of view, whether this mechanism could account for the information flow needed to solve the paradox. That is to say, whether the entropy associated with the different transitions from a given state to the ground state (total evaporation) is comparable with the information the black hole has subtracted from the environment. In order to estimate this, let us assume that the black hole is in an eigenstate of event horizon area $|A, x\rangle$, where $x$ stands for the set of quantum numbers accounting for the corresponding degeneracy $e^{\frac{A}{4}}$ for a given $A$. Now, the transition probability from level $|A, x\rangle$ to $\left|A^{\prime}, x^{\prime}\right\rangle$, for any $x$ and $x^{\prime}$, must be proportional to the ratio between the degeneracy of the levels in question. Accordingly, the probability of transition of going from level $A$ to $A^{\prime}$ cannot strongly depend on whether the transition occurs directly or if it proceeds through intermediate states. The reason is that in order to estimate the transition probability from the initial to the final state in the case of cascading, we have to multiply all the intermediate transition probabilities, assuming that these are statistically independent. After multiplying all these probabilities and cancelling out the intermediate degeneracies, we end up with the ratio between the degeneracies of the final and initial states, exactly as if the transition had occurred in one step. Thus, in order to obtain an estimation of the information that could be transferred to the environment by means of the black-hole spectral lines, should they exist, we assume that all transitions are equally probable. Assume now that the black hole is in its n-th excited state. Then, the decay to the ground state through $k$ intermediate states can occur in $\frac{n !}{k !(n-k) !}$ different ways. Summing over $k$ gives the number of possible different transitions $N_{\text {transitions }}=2^{n}$. Thus the corresponding information capacity is approximately

$$
I_{\text {transitions }} \approx n \ln 2 \propto S_{\mathrm{bh}}
$$

Therefore, the mechanism proposed in 17] could be behind the resolution of the paradox, because enough information could be encoded in the transitions 
to the ground state. Nevertheless, if the Hawking radiation were exactly thermal, then this mechanism would be irrelevant because it lacks the vehicle necessary to transmit the information to a distant observer. However, it has recently been shown, based on information theoretic premisses [18], that the fact that the black hole absorptivity is not unity could render this radiation the intermediary between the black hole and a distant observer. This is so because the radiation is not exactly thermal, i.e. not completely random, and there is enough thermodynamical room in the radiation to transfer all this information.

For an observer far away from the black hole, the situation would be quite acceptable. The black hole appears as a quantum object emitting Hawking radiation whose spectral lines can be used to reconstruct all the information. The black hole is in some sense not very different from an atom. But, contrary to the case of an atom, we can move in closer and investigate the macroscopic black hole and its horizon in greater detail. Then we will observe effects that we will experience as non-local, transmitting information from the interior across the apparent horizon. It is important to note, and this is precisely what we have proven quite generally in the previous section, that this occurs throughout the history of the evaporating black hole. Even when it is macroscopic. There is no way, unless we consider alternative II above, to delay this to the later stages of the evaporation.

The key question is: Can such processes be harmless without causing new paradoxes? In this context we must examine also in a more quantitative way how restrictive the presence of an apparent horizon is. Even if, as we have argued, complete reflection of information at the macroscopic apparent horizon is impossible, it is conceivable that it could take place at the event horizon, which might be as small as the Planck scale and, therefore, sensitive to quantum gravity effects. The key question is whether this is too late, in the sense that the remaining energy would be compatible with the information content. It is commonly accepted that this is really too late. This is also the reason why we have been forced to consider non-local effects. However, a more quantitative analysis would clearly be needed to rule out this possibility, which otherwise would make these effects unnecessary, or at least present only close to the event horizon and the singularity. In fact, through redshifting, Planck scale physics near the event horizon will be magnified tremendously in the eyes of an observer at infinity. While the time to fall into the blackhole is very short for the freely falling black-hole explorer, it would take of 
the order of the whole evaporation time according for an observer at infinity. A Planck time before the event horizon might be well in advance of the complete evaporation, while the black hole is still macroscopic as viewed from the outside. A similar suggestion has been made in [19 in the context of two-dimensional dilaton gravity.

\section{Summary}

Our discussion points out that if we do not allow for non-unitarity, we must either learn to live with an infinite number of metastable or stable black-hole remnants, or there must exist non-local information transfer, which is at work throughout the evaporation, even when the black hole is macroscopic. Our conclusion is that quantum correlations are insufficient to solve either of these problems. In the first case, we have shown that the information storage in correlations does not allow us to decrease the number of needed black-hole states. In the second case, it is well known that EPR correlations do not allow for the kind of information transfer that is needed. If we say that a black hole is like an atom with information encoded in its spectral lines, we still need to confront the issue of locality.

\section{Note Added}

After completion of this work we received a paper [20], where the information paradox is discussed.

\section{Acknowledgments:}

M.S. is partially supported by World Laboratory. M.S. is particulary indebted to Jacob Bekenstein, Maurizio Gasperini and Don Page for enlightening conversations.

\section{References}

[1] S. Hawking, Phys. Rev. D14 (1976) 2460. 
[2] G. 't Hooft, Nucl. Phys. B335 (1990) 138.

[3] C.G. Callan, S.B. Giddings, J.A. Harvey, A. Strominger, Phys. Rev. D45 (1992) R1005.

[4] W. K. Wootters and W. H. Zurek, Nature 299 (1982) 802.

[5] S. Coleman, J. Preskill and F. Wilczek, Nucl. Phys. B378 (1991) 175.

J. Ellis, N.E. Mavromatos and D.V. Nanopoulos, Phys. Lett. B267 (1991) 465 .

[6] J. Preskill, "Do Black Holes Destroy Information?", Proceedings of the International Symposium on Black Holes, Membranes, Wormholes and Superstrings, Texas, January 1992.

[7] T. Banks, A. Dabholkar, M. R. Douglas and M. O'Loughlin, Phys. Rev. D45 (1992) 3607.

[8] S.B. Giddings, "Constraints on Black Hole Remnants", Santa Barbara preprint UCSBTH-9308, hepth/9304027.

[9] F. Wilczek, "Quantum Purity at a Small Price: Easing a Black Hole Paradox", IASSNS-HEP-93/12, hepth/9302096.

[10] W. H. Zurek "information transfer in Quantum Measurements: Irreversibility and Amplification" in Quantum Optics, Experimental Gravitation and Measurement Theory, Eds: P. Meystre and M. O. Scully, (Plenum Press, New York, 1983)

[11] L. D. Landau and E. M. Lifshitz, Statistical Physics (Addison-Wesley, Reading, Mass., 1970).

[12] D. N. Page, Phys. Rev. D13 (1976) 198.

[13] D. N. Page, Phys. Rev. D14 (1976), 3260.

[14] M. Schiffer, "Is it possible to recover information from the black hole radiation?", preprint CERN-TH 6811/93, February 1993 hep-th/9303011.

[15] C.H. Bennett, G. Brassard, C. Crepeau, R. Jozsa, A. Peres and W.K. Wootters, Phys. Rev. Lett. 70 (1993) 1895. 
[16] V. F. Mukhanov, JETP Lett. 44 (1986) 63.

[17] J. G. Bellido "Quantum Black Holes" Stanford University preprint SUITP-93-4, IEM-FT-68/93, hep-th/9302127.

[18] J. D. Bekenstein, "How fast does information leak out from black hole?" Santa Barbara preprint UCSB-TH 93-02, January 1993.

[19] K. Schoutens, H. Verlinde, E. Verlinde, "Quantum Black- Hole Evaporation", Princeton preprint PUPT-1395, IASSNS-HEP-93/25, hepth/9304128

[20] D. N. Page, " Black Hole Information", preprint Alberta-Thy-23-93, hep-th/9305040. 